\title{
Health-Beneficial Properties of Black Sea Shellfish for the Bulgarian Consumers
}

\section{Abstract}

Marine shellfish is considered high-protein, low-fat and low-saturated-fat food, yet providing significant amounts of essential lipids such as polyunsaturated fatty acids (PUFA), phospholipids, fat-soluble vitamins ( $\mathrm{A}_{2} \mathrm{D}_{3}$ and E) and carotenoids. These nutrients exhibit a variety of beneficial effects on human health, therefore the increased intake of marine shellfish could contribute significantly to a diverse and nutritious diet. One of the most consumed Black Sea shellfish species in Bulgaria is the black mussel (Mytilus galloprovincialis). The aim of the present study was to assess health-beneficial properties of Black Sea mussels based on their nutritional lipid composition.

Total lipids (TL) were extracted according to Bligh \& Dyer. Lipid classes were separated by column and thin layer chromatography. Fatty acid composition was determined by GC/MS system and sterol - by GC/FID. Fat-soluble vitamins and carotenoids were analyzed simultaneously using RP-HPLC system.

Mytilus galloprovincialis were characterized by low lipid content: $1.8 \pm 0.08 \mathrm{~g} .100 \mathrm{~g}^{-1} \mathrm{ww}$, more than $50 \%$ representing polar lipids. Sterols were minor components (6.2\% of TL fraction). Fatty acid (FA) composition showed similar distribution: polyunsaturated $>$ monounsaturated $>$ saturated FA in TL, neutral lipids (NL) and polar lipids (PL) classes. Sum of omega-3 (n-3) PUFA was from $47.1 \%$ in TL, $49.2 \%$ in NL and $62.5 \%$ in PL. Ratio n-6/n-3 was 0.2 . Analyzed mussels contained EPA + DHA 0.360 g. $100 \mathrm{~g}^{-1}$ ww. Sterol fraction consisted mainly of cholesterol $(36.3 \%)$ and brassicasterol $(22.9 \%)$. Fat-soluble vitamins contents were: $50.1 \pm 2.01 \mu \mathrm{g} .100 \mathrm{~g}^{-1}$ ww (vitamin A), $7.3 \pm 0.2 \mu \mathrm{g} .100 \mathrm{~g}^{-1}$ ww (vitamin $\mathrm{D}_{3}$ ) and $4500 \pm 140 \mu \mathrm{g} .100 \mathrm{~g}^{-1}$ ww (vitamin E); and carotenoids: $40.1 \pm 1.9 \mu \mathrm{g} .100 \mathrm{~g}^{-1}$ ww (astaxanthin), $490 \pm 5.2 \mu \mathrm{g} .100 \mathrm{~g}^{-1}$ ww ( $\beta$-carotene).

Strong correlation between seafood consumption and various obesity-related disorders can suggest the unique potential of seafood, such as shellfish for supplementation nutrients deficiencies. Recent studies suggest that seafood consumption in Bulgaria is low, increasing the concern of nutrient deficiency, especially PUFA, phospholipids and vitamin $\mathrm{D}_{3}$. This study presented that although Black Sea shellfish is low in TL content, it is a very good source of PL bonded n-3 PUFA $\left(>140 \%\right.$ of RDI), vitamin $\mathrm{D}_{3}(>50 \%$ of RDI) and other essential lipid antioxidants (astaxanthin). The PUFA/SFA (1.4) and n-6/n-3 (0.2) showed the good nutritional quality of Black Sea Mytilus galloprovincialis tissue. Black Sea shellfish lipids are rich in molecules with health-beneficial properties and high potential for developing functional foods and dietary supplements. Increased intake of shellfish should be encouraged to provide essential nutrients to avoid nutritional deficiencies in Bulgarian population.

\section{Acknowledgements}

Bulgaria National Science Fund - №КП-06-ОПР03/11 from 18.12.2018

\section{Conflict of Interest}

There is no conflict of interest 